\title{
Alte und Neue Musik in Praxis und Lehre an der Universität und Musikhochschule in Freiburg im Breisgau
}

\author{
Peter Andraschke \\ Univerza Justusa Liebiga v Giessnu \\ Justus-Liebig-Universität Giessen
}

Die Einflüsse der Leipziger Musiktradition auf die Initiativen der historischen Musikwissenschaft und Praxis in Freiburg i. Br. sind unverkennbar. Von der Universität und der Musikhochschule in Freiburg gingen aber zahlreiche eigene Anregungen für die Musik des Mittelalters, des Barock und für die Neue Musik im 2o. Jahrhundert aus. Das möchte ich an einigen Beispielen darstellen, die belegen, daß für ein Studium der Musik die wissenschaftliche Seite nicht eine ausschließliche Domäne der Universität sein sollte und die Praxis nur eine der Musikhochschulen bzw. Konservatorien.

1457 gründete Erzherzog Albrecht VI. von Österreich in Freiburg, das damals zu Vorderösterreich gehörte, die heutige Albrecht-Ludwigs-Universität, die damit nach Wien die zweite habsburgische Gründung ist. Als Freiburg mit dem Breisgau 1805 an Baden fiel, sicherte der Großherzog Ludwig von Baden ihren Erhalt und wurde als zweiter Name aufgenommen.

Wilibald Gurlitt (1889-1963), Sohn des Kunsthistorikers Cornelius Gurlitt, promovierte 1914 bei Hugo Riemann in Leipzig mit einer Arbeit über Michael Praetorius (1560-1629). Er kam 1918 als Lektor an die Freiburger Universität und gründete 1920 das Musikwissenschaftliche Seminar. Sein Interesse galt einer praxisorientierten Ausrichtung des Fachs. So gehörte zu seinen Aktivitäten auch das Sammeln historischer Musikinstrumente. ${ }^{1}$ Das von ihm ins Leben gerufene Collegium musicum machte

1 Markus Zapf, „'Das Orchester des 16. und 17. Jahrhundert in seinem originalen Klangwesen erwecken'. Die Sammlung der historischen Musikinstrumente des Musikwissenschaftlichen Seminars Freiburg im Breisgau“, in Musik in Baden-Württem- 
mit deutschlandweiten Konzerten, z.B. 1922 in Karlsruhe und 1924 in Hamburg, erstmals eine breitere Öffentlichkeit mit der Musik des Mittelalters bekannt.

Die nach den Dispositionen in der Organographia (Syntagma musicum, Bd. 2, 1619) von Michael Praetorius konzipierte Orgel ließ Gurlitt von dem Ludwigsburger Oscar Walcker für den Hörsaal nachbauen und initiierte damit neue Anregungen für die deutsche Orgelbewegung der 1920er Jahre. Die Orgel wurde 1944 durch Bomben zerstört und 1954/55 von Werner Walcker-Mayer mit mitteltöniger Stimmung wieder errichtet.

Auch Hermann Erpf (1891-1969) hatte bei Riemann in Leipzig promoviert und war von 1923 bis 1925 Lektor für Musiktheorie am Freiburger Institut. Zunächst unter Gurlitt und vor allem unter Erpf als neuem Leiter, widmete sich das Collegium verstärkt der Neuen Musik. Die öffentlich zugänglichen Konzerte wurden ausschließlich von einheimischen Kräften gestaltet, darunter Studenten und Musikliebhaber. Die Einführungen gab meist Erpf.

Diese Veranstaltungen, so Erpf,

gingen aus dem Bedürfnis hervor, das Musikschaffen der Gegenwart in seinen Haupterscheinungen fortlaufend kennen zu lernen. Ausgehend von der späten Kammermusik Max Regers, wurden in strenger Beschränkung auf diese heute führende Gattung, die hervortretenden Namen, zunächst des deutschen Sprachgebiets, vorgeführt; ein einleitender Vortrag orientierte jeweils über Komponisten und Werke. [...] In jedem Semester fanden durchschnittlich vier auch öffentlich zugängliche Abende im Hörsaal des Musikwissenschaftlichen Seminars, Bertholdstr. 14, statt. Sämtliche Aufführungen waren für Freiburg erstmalig, einige davon Uraufführungen; schwer verständliche Werke wurden mehrfach wiederholt. [...] Zum erstenmal wurde hier die zeitgenössische Musik in den Rahmen der sog. Collegia musica, die nach Hugo Riemanns Vorbild ihre Hauptaufgabe in dem Studium und der Pflege alter Musik sehen, und damit als Gesamterscheinung in das Arbeitsgebiet eines musikwissenschaftlichen Universitätsseminars einbezogen. ${ }^{2}$

berg, Bd. 11, hrsg. von. Georg Günther, Walter Salmen und Gabriele Busch-Salmen (München: Strube -Verlag, 2004), 187-219.

2 Informationsblatt mit einem „Verzeichnis der bisherigen Veranstaltungen [7. 11. 1921-23. 7. 1923]“. 
Für seinen ersten Vortrag am 29. Mai 1922 wählte Erpf die beiden ersten Sätze von Arnold Schönbergs Streichquartett op. 10, wobei der zweite Satz wiederholt wurde. Am 12. Februar 1923 stellte er Ernst Kreneks Streichquartett op. 6 und Egon Wellesz' 4. Streichquartett op. 28 vor. Für das Hindemith-Programm am 6. Mai, in dem die Sonate für Cello solo op. 25 Nr. 3 uraufgeführt wurde, hatte man das Amar-Quartett, in dem Hindemith als Bratschist mitwirkte, verpflichten können. Man bot mithin vielfältige Programme, die offen für alle Musikströmungen der Zeit waren. Um eine größere Öffentlichkeit für die zeitgenössische Musik zu interessieren, gliederte Erpf die Konzerte in die von Ewald Lindemann, dem neuen Kapellmeister des Stadttheaters gegründete Arbeitsgemeinschaft für neue Musik ein.

In einem ausführlichen Artikel im Januar/Februar-Heft 1927 von Pult und Taktstock mit dem Titel Erziehung zum Verständnis zeitgenössischer Musik ${ }^{3}$ stellte Erich Katz das Engagement für Neue Musik in Freiburg i. Br. als Vorbild und Anregung für andere Städte vergleichbarer Größenordnung vor:

Freiburg, eine mittelgroße Universitätsstadt mit einem Theater von gutem Durchschnitt und regsamen Konzerunternehmungen, unterschied sich im übrigen in nichts von dem Charakter und der Tätigkeit zahlreicher ähnlicher Städte. Der erste Anstoß zu einer bewussten Pflege zeitgenössischer Musik ging, ein seltener und darum um so rühmlicherer Fall, von der Universität aus. Wilibald Gurlitt, der seit 1919 dort wirkende Professor der Musikwissenschaft, begann damit, in seinem Collegium Musicum regelmäßige Vorträge mit Vorführungen moderner Musik zu bringen; die Berufung eines Lektors für Musiktheorie und moderne Musik [...] Hermann E $r p f$, der seit dem Frühjahr die Leitung dieser modernen Collegia Musica übernahm, ermöglichte es, das Ganze auf eine breitere Basis zu stellen. So wurden, neben ständigen Vorlesungen und Übungen über Probleme und Gegenstände neuer Musik, in den Jahren 1921 bis 1923 etwa 20 öffentliche Veranstaltungen gebracht, immerhin zu einer Zeit, da den mittleren Schönberg zu spielen noch ein Wagnis war, das oft mit Zischen und Pfeifen belohnt wurde; da ein Komponist wie Krenek eben erst, entdeckt', andere [...] noch weit vor ihrer Entdeckung standen. [...] So wurde in einer segensreichen

3 Erich Katz, „Erziehung zum Verständnis zeitgenössischer Musik (Die Freiburger ,Arbeitsgemeinschaft für neue Musik')“, in Pult und Taktstock 4 (Januar/Februar 1927): 6-11. 
Vorarbeit, deren festes Fundament über die anfangs recht erheblichen Widerstände hinwegzutragen vermochte, der Boden zum erstenmal bereitet; und als dann 1924 ein junger und selbst von den Ideen der neuen Kunst erfüllter Kapellmeister, Ewald Lindemann, zum musikalischen Oberleiter des Theaters und der Stadt berufen wurde, da ergab es sich sehr bald, daß eine Verbindung entstand, deren Produkt die Freiburger, Arbeitsgemeinschaft für Neue Musik' wurde. ${ }^{4}$

Für die Musikpflege an den Universitäten sind auch die verschiedenen Orchester und Chöre wichtig. Besonders in Leipzig hat die Universitätsmusik eine lange Tradition, die bis zu ihrer Gründung im Jahre 1409 zurückreicht. So wurden im 16. Jahrhundert Vorlesungen und Übungen von Kantoren der Thomas- und Nikolaikirche zur Ausbildung von praktischen Musikern durchgeführt. Im 17. Jahrhundert kam es zur Bildung einzelner studentischer Instrumentalmusikensembles, sogenannten Collegia musica. Ein solches gründete z.B. 1701 der damalige Jurastudent Georg Philipp Telemann. In dieser Tradition stand Hugo Riemanns 1908 mit der Gründung des Musikwissenschaftlichen Instituts Collegium musicum. Die Tradition der Chor- und Orchesterpflege an der Universität Freiburg setzte erst spät ein. Heute existieren mehrere instrumentale Gruppen und Chöre, deren anfangs private Initiativen später häufig von der Universitätsverwaltung unterstützt wurden. Ein Beispiel ist der Russische Chor, der von dem 1897 im damaligen Sankt Petersburg geborenen Alexander Kressling 1930 gegründet wurde und bis heute aktiv ist. Sein Repertoire sind Lieder aus dem alten Russland und das Liedgut der Altgläubigen. Kressling kam 1917 als Emigrant nach Freiburg und wurde Lektor für Russisch. Heute existiert eine breite Palette von Chor- und Instrumentalensembles an der Universität.

Ein Konservatorium gab es in Freiburg seit 1898. Erst nach dem 2. Weltkrieg wurde von Gustav Scheck (1901-1984) in Verbindung mit Gurlitt die Musikhochschule gegründet: 1946 zunächst städtische Einrichtung, wurde sie 1948 vom Staat übernommen. Schwerpunkte waren die Vermittlung zeitgenössischer Musik und die Pflege einer historisch fundierten Aufführungspraxis. Das zeigt bereits die Berufung des ersten Lehrkörpers. Die folgenden Beispiele beschränken sich auf die Zeit bis etwa Mitte der 197oer Jahre. 
Scheck, ein bekannter Flötist, hatte in Freiburg Flöte und daneben Musikwissenschaft bei Gurlitt und Erpf studiert und erhielt hier sein erstes Engagement am Stadttheater. Zuletzt war er 1934-1945 Professor an der Berliner Musikhochschule gewesen. Bekannt ist er vor allem durch seinen Einsatz für die Belebung einer quellenorientierten Barockmusik geworden. Er konzertierte u.a. mit dem 1930 von ihm und dem Schweizer Gambisten und Cellisten August Wenziger (1905-1996) gegründeten Kammermusikkreis Scheck-Wenziger, dem ersten Barockensemble mit originalen Instrumenten und von 1935-65 zusammen mit Fritz Neumeyer (1900-1983) und Wenziger mit dem Kammermusiktrio für Alte Musik. Scheck war zudem Interpret der gemäßigten Moderne. So wurden ihm mehrere Kompositionen gewidmet, z.B. von Genzmer je zwei Flötenkonzerte und Sonaten und von Wolfgang Fortner (1907-1987) die Sonate für Flöte und Klavier (1947).

Scheck berief 1946 (-1968) Neumeyer als Professor für historische Tasteninstrumente. Von Curt Sachs, dem Direktor des Berliner Instrumentensammlung 1928 angeregt, begann er 1930 selbst mit dem Sammeln von historischen Tasteninstrumenten ${ }^{5}$ und dem Erforschen der Quellen ihrer Aufführungspraxis. 1933 gründete er die Saarbrücker Vereinigung für Alte Musik. Er war als Solist begehrt und wurde Mitglied renommierter historisch besetzter Ensembles, u.a. seit 1954 der Capella Coloniensis und 196065 der Wiener Solisten. Von Neumeyer existieren zahlreiche Tonträger.

Die Pianistin und Cembalistin Edith Picht-Axenfeldt (1914-2001) kam 1947 an die Hochschule. Sie ist als Bach- und Chopininterpretin bekannt geworden, widmete sich aber schon früh der zeitgenössischen Musik. So spielte sie den Cembalopart bei der Uraufführung von Hans Werner Henzes Apollo et Hyazinthus (1948) und konzertierte mit Carl Seemann in Bartóks Sonate für zwei Klaviere und Schlagzeug (1937). Sie hatte auch Schönbergs Klavierwerk im Repertoire. Unter den zahlreichen ihr gewidmeten Kompositionen sind Isang Yuns Cembalostück Shao Yang Yin (1966, UA 1968) und Heinz Holligers Dunkle Spiegel für Vokalquintett, Bariton und 5 Instrumentalgruppen (1996).

Auch der Pianist Carl Seemann (1910-1983) gehörte zu den ersten Professoren der Musikhochschule und wurde von 1964-1974 ihr Direktor. Er ist vor allem durch seine Mozartinterpretationen bekannt geworden, auch als Duopartner des Geigers Wolfgang Schneiderhahn. Zudem setzte er sich für

5 Die Sammlung befindet sich heute im Schloß von Bad Krozingen, siehe Susanne Berkemer und Markus Zapf, Hrsg., Fritz Neumeyer und seine Sammlung (Freiburg i. Br.: Rombach, 2014). 
die gemäßigte Moderne ein und spielte z.B. die Uraufführung von Fortners Mouvements für Klavier und Orchester (1953).

Als stellvertretenden Direktor holte Scheck 1946 Harald Genzmer (1909-2007), einen Schüler von Paul Hindemith, der als Komponist und Lehrer dessen die Tradition fortsetzte. Er wechselte 1957 an die Hochschule in München. Sein Nachfolger wurde von 1967-1973 Wolfgang Fortner (1907-1987). Fortner hatte in Leipzig neben Komposition am Konservatorium auch Philosophie und Musikwissenschaft an der Universität belegt. Er wurde einer der renommiertesten Kompositionslehrer nach dem Zweiten Weltkrieg ${ }^{6}$ und ein einflußreicher Musikorganisator. Bei den Darmstädter Ferienkursen war er von Anfang an als Komponist und Lehrer engagiert, übernahm 1963-1978 die von Karl Amadeus Hartmann (1905-1963) ins Leben gerufene Konzertreihe Musica viva in München. Mit seinen Schülern (darunter Musikwissenschaftler und Dirigenten) deckte er alle Aspekte der Moderne ab (in Freiburg u.a. Milko Kelemen, Nam Jun Paik, Wolfgang Rihm, Hans Zender). Er gründete das Institut für Neue Musik an der Hochschule, dessen öffentliche Konzerte vor allem von Studenten und Kollegen der Hochschule gestaltet wurden und auch ein Podium für die Werke seiner Schüler boten. Seine Seminare, an denen auch interessierte Studenten des Musikwissenschaftlichen Instituts teilnahmen konnten, boten Analysen der Musik seit dem Mittelalter (z.B. Guillaume Machaut), die der eingeladenen Komponisten (u.a. Luigi Nono, Isang Yun) gaben persönliche Einblicke in die aktuelle Musikszene.

Der Musikwissenschaftler und Pädagoge Erich Doflein (1900-1977) promovierte bei Max Schneider an der Breslauer Universität und ging danach nach Freiburg, um seine Studien bei Gurlitt und Erpf weiterzuführen. 1928 war er hier Mitbegründer eines privaten Instituts für Musiklehrer, da eine Musikhochschule noch fehlte. Bereits in den 1920er Jahren galt er als angesehener Kritiker für zeitgenössische Musik und schrieb u.a. für die Freiburger Zeitung, die Frankfurter, den Anbruch und Melos. Mit seiner Frau Elma, einer Geigerin, gab er das mehrbändige Geigenschulwerk heraus, eines der bedeutendsten Werke der modernen Violinpädagogik. Er regte dafür u.a. Béla Bartók zu den 44 Duos für 2 Violinen (1931) an, von denen er einige in die Geigenschule aufnehmen konnte. 1948 initiierte er das Institut für Neue Musik und Musikerziehung in Bayreuth, das bald

6 Matthias Roth, Ein Rangierbahnhof der Moderne. Der Komponist Wolfgang Fortner und sein Schülerkreis 1931-1986: Erinnerungen, Dokumente, Hintergründe Porträts (Freiburg i. Br./Berlin/Wien: Rombach Verlag, 2008).

75 Bände (Mainz: Schott Musik 1932-1950), ins Englische übersetzt, ebda. 1957. 
nach Darmstadt übersiedelte und war 1956 bis 1960 dessen Vorsitzender. Er gehört zu den Gründungsdirektoren der Staatlichen Musikhochschule und war bis 1965 Professor. ${ }^{8}$ Sein Spezialgebiet war die zeitgenössische Moderne. Für die von ihm vertretenen Ansichten ist der kommentierte Briefwechsel mit Theodor W. Adorno aufschlußreich. ${ }^{9}$

Der Schweizer Heinz Holliger (1939) ist als Oboist, Komponist ${ }^{10}$ und Dirigent weltweit bekannt. Als Oboist entwickelte er in Zusammenarbeit mit Komponisten neue Spieltechniken, erhielt Widmungen bekannter Komponisten und spielte zahlreiche Uraufführungen. Er hatte einen wichtigen Anteil bei der Wiederentdeckung der Musik von Jan Dismas Zelenka (1679-1745) durch die Einspielung der 6 Triosonaten für die DGG Archivproduktion im Jahr 1972, die er in den Archiven entdeckt hatte, ebenso bei der Aufnahme von dessen Orchesterwerken. An der Freiburger Musikhochschule wirkte er als Professor für Oboe.

Das Freiburger Beispiel zeigt das übergreifende Zusammenwirken von Theorie und Praxis zwischen Universität und Musikhochschule und auch anderen Instituten. Diese erfolgreichen Ansätze sollten Standard sein und ausgebaut werden. Die universitäre Musikwissenschaft darf sich nicht ausschließlich auf die theoretische Forschung und Lehre beschränken, sondern in einem vertretbaren Maß auch die Praxis einbeziehen. Z.B. was das Mittelalter betrifft: den Studierenden den Choral und die darauf aufbauende Mehrstimmigkeit durch das Singen, und zwar nach Originalen und nicht nach Übertragungen, klanglich näher bringen und dadurch die verschiedenen Notationen und ihren Einfluß auf die Kompositionsart vermitteln. Oder, was die Neue Musik betrifft: die Schwierigkeiten und Probleme einer Verwirklichung des Notenbildes (Zwölfton-Partituren, Aleatorik, graphische Notation usw.) praktisch erleben lassen. Die Musikhochschulen bzw. Konservatorien auf der anderen Seite sollten bei ihrer Ausbildung nicht alleine die virtuose Seite im Blick haben, sondern auch die histori-

Lars Ulrich Abraham, Hrsg., Erich Doflein. Festschrift zum 7o. Geburtstag (Mainz: Schott, 1972).

9 Andreas Jacob, Hrsg., Theodor W. Adorno - Erich Doflein. Briefwechsel. Mit einem Rundfunkgespräch und 3 Aufsätzen Erich Dofleins (Folkwangstudien 2) (Hildesheim: Olms, 2006); siehe hier auch die Einleitung von Andreas Jacob, „Der Briefwechsel Adorno-Doflein. Zwei Perspektiven auf das westdeutsche Musikleben der Nachkriegszeit“.

10 Peter Andraschke, „Dichtung in Musik. Stockhausen, Trakl, Holliger“, in Stimme und Wort in der Musik des 2o. Jahrhunderts (=Wiener Schriften zur Stilkunde und Aufführungspraxis), Bd. 1 (der Sonderreihe Symposien zu WIEN MODERN), hrsg. von Hartmut Krones (Wien/Köln/Weimar: Böhlau, 2001), 341-55. 
sche Aufführungspraxis vermitteln, sei es in einem eigenen Institut, besser auch durch entsprechend interessierte und geschulte Instrumental- und Gesangslehrer.

\section{Bibliographie}

Abraham, Lars Ulrich, Hrsg. Erich Doflein. Festschrift zum 7o. Geburtstag. Mainz: Schott, 1972.

Andraschke, Peter. „Dichtung in Musik. Stockhausen, Trakl, Holliger“. In Stimme und Wort in der Musik des 2o. Jahrhunderts (=Wiener Schriften zur Stilkunde und Aufführungspraxis). Sonderreihe Symposien zu WIEN MODERN, Bd. 1, hrsg. von Hartmut Krones, 341-55. Wien/Köln/Weimar: Böhlau, 2001.

Berkemer, Susanne und Markus Zapf, Hrsg. Fritz Neumeyer und seine Sammlung. Freiburg i. Br.: Rombach, 2014.

Doflein, Erich und Elma. Das Geigen-Schulwerk, Bd. 1-5. Mainz: Schott Musik, 1932-1950.

Jacob, Andreas, Hrsg. Theodor W. Adorno - Erich Doflein. Briefwechsel. Mit einem Rundfunkgespräch und 3 Aufsätzen Erich Dofleins (Folkwangstudien 2). Hildesheim: Olms, 2006.

Jacob, Andreas. „Der Briefwechsel Adorno-Doflein. Zwei Perspektiven auf das westdeutsche Musikleben der Nachkriegszeit". In Theodor W. Adorno - Erich Doflein. Briefwechsel. Mit einem Rundfunkgespräch und 3 Aufsätzen Erich Dofleins (Folkwangstudien 2), hrsg. von Andreas Jacob. Hildesheim: Olms, 2006.

Katz, Erich, „Erziehung zum Verständnis zeitgenössischer Musik (Die Freiburger ,Arbeitsgemeinschaft für neue Musik')“. In Pult und Taktstock 4 (Januar/Februar 1927): 6-11.

Roth, Matthias. Ein Rangierbahnhof der Moderne. Der Komponist Wolfgang Fortner und sein Schülerkreis 1931-1986: Erinnerungen, Dokumente, Hintergründe Porträts. Freiburg i. Br./Berlin/Wien: Rombach Verlag, 2008.

Zapf, Markus. „'Das Orchester des 16. und 17. Jahrhundert in seinem originalen Klangwesen erwecken'. Die Sammlung der historischen Musikinstrumente des Musikwissenschaftlichen Seminars Freiburg im Breisgau“. In Musik in Baden-Württemberg, Bd. 11, hrsg. von Georg Günther, Walter Salmen und Gabriele Busch-Salmen, 187-219. München: Strube -Verlag, 2004. 